\title{
Dynamical properties of Molecular Cloud Complexes at the Epoch of Reionization
}

\author{
T. K. Daisy Leung ${ }^{1,2} \mathbb{D}^{\text {, Andrea Pallottini }}{ }^{3,4}{ }^{1}$, Andrea Ferrara ${ }^{4,5}$ and \\ Mordecai-Mark Mac Low ${ }^{2,6}$ \\ ${ }^{1}$ Department of Astronomy, Cornell University, NY, USA \\ email: tleung@astro.cornell.edu \\ ${ }^{2}$ Center for Computational Astrophysics, Flatiron Institute, NY, USA \\ ${ }^{3}$ Centro Fermi, Rome, Italy \\ ${ }^{4}$ Scuola Normale Superiore, Pisa, Italy \\ ${ }^{5}$ Kavli Institute for the Physics and Mathematics of the Universe (IPMU), \\ University of Tokyo, Japan \\ ${ }^{6}$ American Museum of Natural History, NY, USA
}

\begin{abstract}
The Atacama Large (Sub-)millimeter Array (ALMA) has provided glimpse of the interstellar medium (ISM) properties of galaxies at the Epoch of Reionization (EoR); however, detailed understanding of their internal structure is still lacking. We present properties of molecular cloud complexes (MCCs) in a prototypical galaxy at this epoch studied in cosmological zoom-in simulations (Leung et al. 2019c). Typical MCC mass and size are comparable to nearby spirals and starburst galaxies $\left(M_{\text {gas }} \sim 10^{6.5} M_{\odot}\right.$ and $\left.R \simeq 45-100 \mathrm{pc}\right)$. MCCs are highly supersonic, with velocity dispersion of $\sigma_{\text {gas }} \simeq 20-100 \mathrm{~km} \mathrm{~s}^{-1}$ and pressure of $P / k_{B} \simeq 10^{7.6} \mathrm{~K} \mathrm{~cm}^{-3}$, which are comparable to gas-rich starburst galaxies. In addition, we perform stability analysis to understand the origin and dynamical properties of MCCs. We find that MCCs are globally stable in the main disk of Althæa. Densest regions where star formation is expected to take place in clumps and cores on even smaller scales instead have lower virial parameter and Toomre- $Q$ values. Detailed studies of the star-forming gas dynamics at the EoR thus require a spatial resolution of $<40 \mathrm{pc}\left(\simeq 0.01^{\prime \prime}\right)$, which is within reach of ALMA, to complement studies of stellar populations at EoR using the James Webb Space Telescope (JWST).
\end{abstract}

Keywords. galaxies: high-redshift - galaxies: ISM, galaxies: evolution, ISM: structure, ISM: kinematics and dynamics ISM: clouds

\section{Introduction}

Early galaxies have higher molecular gas fractions, star formation rate, and smaller sizes than present-day galaxies (e.g., Bouwens et al. 2011; Decarli et al. 2016, 2017; Leung et al. 2019c). As such, they are expected to be significantly more ionized, with more intense and harder interstellar radiation fields. Their metallicity and dust content are also expected to be lower, which in turn affect the thermal and chemical state of the multi-phase ISM. Here, we pose the question: what are the physical properties of molecular cloud structures in early galaxies, and how do they differ from those found in local galaxy populations?

\section{Cosmological Zoom-in Simulations: SerRA}

The simulations used are briefly summarized here (see Pallottini et al. 2017a,b for details). SERRA is a suite of cosmological zoom-in simulations performed using Eulerian hydrodynamics and adaptive mesh refinement (AMR) techniques, covering a comoving 
box of $20 \mathrm{Mpc} h^{-1}$ in size and zooms in on a target halo of mass $M_{\mathrm{DM}} \simeq 10^{11} M_{\odot}$. The Lagrangian region of the halo $\left(2.1 \mathrm{Mpc} h^{-1}\right)$ has a dark matter mass resolution of $\simeq 6 \times 10^{4} M_{\odot}$, and is spatially refined with the finest cell size of $l_{\text {cell }} \simeq 30 \mathrm{pc}($ at $z=6)$, i.e., comparable to the size of local giant molecular clouds. Our model includes a nonequilibrium chemical network (Grassi et al. 2014; Bovino et al. 2016), where abundances are calculated using an on-the-fly non-equilibrium formation of molecular hydrogen scheme (Pallottini et al. 2017b). The main zoom galaxy (Althæa) is a Lyman-break galaxy at $z \simeq 6$, with a stellar mass of $M_{\star} \simeq 3 \times 10^{10} M_{\odot}$, a metallicity of $Z \simeq 0.5 Z_{\odot}$, a molecular gas mass of $M_{\mathrm{H} 2} \simeq 5 \times 10^{7} M_{\odot}$, and a SFR of $30-80 M_{\odot} \mathrm{yr}^{-1}$.

\section{Physical Properties and Stability of $z \sim 6$ Molecular Gas}

The typical size and mass of MCCs are $R \simeq 50 \mathrm{pc}$ and $M_{\text {gas }} \simeq 10^{6.5} M_{\odot}$, comparable to massive molecular structures observed in nearby star-forming and starburst galaxies (e.g., Leroy et al. 2015). MCCs are highly supersonic with an average Mach number of $\overline{\mathcal{M}} \simeq 6$. Their velocity dispersion and gas surface density are systematically higher than Milky Way clouds, but comparable to $z \sim 2$ starburst galaxies (e.g., Swinbank et al. 2011). High pressure $\left(\bar{P} \simeq 10^{7.6} \mathrm{~K} \mathrm{~cm}^{-3}\right)$ MCCs are found throughout the disk of Althæa and result from extra-planar flows and high velocity accretion/SN-driven outflows.

We also perform virial analysis, as motivated by observations, to assess the stability of MCCs. Virial parameter is lowest for MCCs in the densest regions, some of which are located in regions with Toomre $Q_{\text {eff }} \lesssim 1$. These MCCs are unstable against collapse, where star formation is expected to take place within their gas clumps and cores on scales $\lesssim 40$ pc as energy quickly dissipates.

Contribution from the stellar component plays an important role in governing the stability of the MCCs against axisymmetric perturbations $\left(Q_{\text {eff }}\right)$, especially in the central part of Althæa. Similarly, stabilizing effect due to the thickness of its disk is also nonnegligible. This illustrates the importance of accounting for both effects when examining the stability of molecular gas structures in relatively evolved and enriched systems at high redshift that are preferentially being observed now.

\section{Summary and Outlook}

We study the origin and dynamical properties of MCCs in prototypical galaxies at the EoR in numerical simulations to provide a framework within which upcoming observations can be compared against to aid in the interpretation. Details of our findings are reported in Leung et al. (2019c). Concerning the topic of this symposium, our results imply that spatially resolution better than $\simeq 40 \mathrm{pc}$ are needed to examine the truly starforming structures, and thus, star formation in the first galaxies. Such resolution is within reach of ALMA and will complement studies of stellar population in the first galaxies using JWST.

\section{References}

Bouwens, R. J., Illingworth, G. D., Labbe, I., et al. 2011, Nature, 469, 504

Bovino, S., Grassi, T., Capelo, P. R., Schleicher, D. R. G., \& Banerjee, R. 2016, A\& A, 590, A15

Decarli, R., Walter, F., Aravena, M., et al. 2016, ApJ, 833, 69

Decarli, R., Walter, F., Venemans, B. P., et al. 2017, Nature, 545, 457

Grassi, T., Bovino, S., Schleicher, D. R. G., et al. 2014, MNRAS, 439, 2386

Leroy, A. K., Bolatto, A. D., Ostriker, E. C., et al. 2015, ApJ, 801, 25

Leung, T. K. D., Riechers, D. A., Baker, A. J., et al. 2019a, ApJ, 871, 85

Leung, T. K. D., Pallottini, A., Ferrara, A., \& Mac Low, M.-M. 2019c, ApJ, Submitted

Pallottini, A., Ferrara, A., Bovino, S., et al. 2017a, MNRAS, 471, 4128

Pallottini, A., Ferrara, A., Gallerani, S., et al. 2017b, MNRAS, 465, 2540

Swinbank, A. M., Papadopoulos, P. P., Cox, P., et al. 2011, ApJ, 742, 11 